\title{
Las concepciones ontológicas como punto de acceso a las ciencias sociales y sus diversas perspectivas metodológicas
}

\section{The ontological conceptions as an access point to the Social Sciences and their diverse methodological perspectives}

\author{
Luis Diego Soto Kiewit \\ Doctorado en Ciencias Sociales, Universidad Nacional, Costa Rica \\ luis.soto.kiewit@una.cr
}

Recibido: 24/09/2019 • Aceptado: 25/08/2020

\begin{abstract}
Resumen
Este artículo propone la lectura de las concepciones ontológicas como una forma de acercamiento y acceso a las distancias y discrepancias de las diversas perspectivas de las ciencias sociales. El objetivo es identificar cómo las distancias epistemológicas y metodológicas pueden ser entendidas desde la noción de realidad en la que se sustentan las propuestas de conocimiento. La reflexión incluye un diálogo con dos perspectivas metodológicas de las ciencias sociales, esto con el objetivo de ejemplificar cómo los fundamentos ontológicos permiten comprender afirmaciones o posturas en niveles reflexivos más abstractos, que acá llamaremos: niveles superiores. Para este ejercicio demostrativo se toma en cuenta al estructuralismo constructivista y posestructuralismo.
\end{abstract}

Palabras Clave: Ciencias sociales, perspectivas metodológicas, ontología, estructuralismo constructivista, posestructuralismo. 


\begin{abstract}
This article proposes the reading of ontological conceptions as a way of approaching and accessing the distances and discrepancies of the different perspectives of the social sciences. The objective is to identify how epistemological and methodological distances can be understood from the notion of reality on which knowledge proposals are based. The reflection includes a dialogue with two methodological perspectives of the social sciences, this to exemplify how the ontological foundations allow us to understand affirmations or positions in more abstract reflective levels, which we will call here: higher levels. For this demonstrative exercise, constructivist and poststructuralism structuralism are considered.
\end{abstract}

Key words: Social Sciences, Methodological Perspectives, Ontology, Structuralism constructivist, Poststructuralism.

\title{
INTRODUCCIÓN
}

En estas breves líneas y en respuesta a la pregunta: ¿Cómo entender las perspectivas metodológicas ${ }^{1}$ de las ciencias sociales?, se proponen dos ideas: la primera explicar por qué la ontología es una alternativa para el abordaje y diferenciación de los enfoques y disciplinas de este campo (en toda su diversidad); la segunda, identificar, en las perspectivas metodológicas seleccionadas, cómo la noción de realidad orienta principios y definiciones centrales de la propuesta de conocimiento.

En las ciencias sociales desde sus orígenes se han posicionado diversidad de propuestas teóricas, epistemológicas y metodológicas. Las posturas implican debates sobre la forma de entender la práctica científica, es decir, cómo abordar los fenómenos de estudio y, se puede decir, que hasta la definición de los problemas de interés.

1 En el texto se emplea de manera indistinta los calificativos perspectiva y corriente, con el objetivo de hacer referencia a las diversas orientaciones metodológicas de las ciencias sociales. Esto porque permiten dejar claro que se trata de líneas en las que se enmarcan diversidad de autores y autoras que, aunque tienen distancias y discrepancias, manejan una serie de valoraciones y definiciones que les hacen coincidir. A la vez, se distancia de conceptos como paradigma que, desde su primera aparición (Kuhn, 1996), carga diversidad de cuestionamientos, así como un uso tergiversado y polisémico, por no mencionar la distancia que tiene de las ciencias sociales (Dogan, 1997a y 1997b). 
Tomando en cuenta lo anterior, un primer acercamiento a la diversidad de perspectivas en las ciencias sociales puede parecer abrumador, ya que son abundantes las líneas de desarrollo, y estas no siempre pasan por un ejercicio de definición que refleje el lugar desde el que se está trabajando. Incluso es posible identificar investigaciones que en la práctica dejan ver poca riguridad epistemológica y metodológica.

En este escenario, se proponen las concepciones ontológicas como un punto de acceso a la discusión de las ciencias sociales, es decir, una vía para la comprensión de las diversas corrientes. Según esto, el nodo central de la discusión no está en las disciplinas y cómo cada una se enfoca en una parcela de la realidad, sino en los principios desde los cuales cada una de ellas y sus diversos enfoques y teorías entienden la realidad y desde ahí proceden en el proceso de conocimiento.

La importancia de la propuesta sintetizada en este artículo reside en tres aspectos; (i) que las concepciones ontológicas posibilitan la identificación de los principios más básicos desde los cuales se organiza cada una de las corrientes de las ciencias sociales; (ii) que permite entender la relación entre los niveles -el axiológico, epistemológico y metodológico- de cada una de las corrientes de las ciencias sociales; (iii) que constituye una herramienta para entender las diferencias a lo interno de las disciplinas y de los diversos enfoques a lo interno de cada una de ellas.

El documento está compuesto por cinco apartados: (i) la introducción, (ii) el apartado central, que esboza la propuesta del artículo, (iii) la contextualización de la discusión sobre las perspectivas de las ciencias sociales, (iv) un subapartado en el que se aplica la propuesta, al abordar dos perspectivas metodológicas (el posestructuralismo y el estructuralismo constructivista), y, por último, (v) las conclusiones generales que sintetizan la reflexión.

\section{PROPUESTA DE DISCUSIÓN}

En el estudio de las ciencias sociales, como perspectivas de conocimiento, se deben tener en consideración distintos niveles, ya que los frentes de discusión son diversos. Acá se retoman los puntos elementales de posicionamiento, es decir, las nociones epistemológicas, axiológicas, metodológicas y ontológicas. Cada uno de estos espacios de definición posibilitan amplias discusiones y perspectivas diferenciadas a lo interno de cada disciplina de las ciencias sociales. 
Con una revisión general de los distintos esfuerzos de definición de las perspectivas metodológicas en las ciencias (García, 2000) o en las ciencias sociales (De la Garza y Leyva, 2012), se puede evidenciar que las discusiones están enfocadas en las distancias o tensiones metodológicas y epistemológicas entre cada una de las concepciones de ciencia.

La propuesta de estas líneas es definir las concepciones ontológicas como punto de acceso al debate de las ciencias sociales (figura 1). El argumento central es que la noción de realidad -concepción ontológica-, desde la que se construye cada perspectiva, es la base sobre la cual se cimientan las definiciones en los otros niveles (como ilustran los vectores de color azul); a manera de derivación, cada nivel tiene implicaciones sobre el siguiente. Esta propuesta supone que la concepción ontológica es el eje desde el cual se soportan los demás niveles.

Lo anterior se basa en la premisa de que todo abordaje de la realidad parte, necesariamente, de una noción, más o menos elaborada, de lo que es esa realidad y la manera en la que se organiza. Ello implica que las concepciones ontológicas estén en la base de toda propuesta de conocimiento de las ciencias sociales y sus diversas disciplinas, teorías y conceptos.

Figura 1. Definición de niveles²

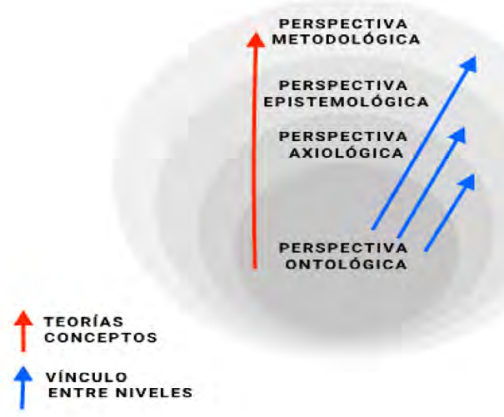

Nota: elaboración propia.

2 La figura ilustra la propuesta que se condensa en el artículo. En ella se coloca como eje central la concepción ontológica (como noción más general) a partir de la cual se soportan los niveles superiores. Esto explica la orientación de los vectores (de color rojo y azul) que tienen como punto de partida la perspectiva otológica. Además, permiten identificar las conexiones entre niveles, esto último muestra que la perspectiva ontológica le da sentido a cada a los niveles superiores. 
Según lo anterior, toda definición conceptual, teórica o paradigmática, está basada en un posicionamiento en cada uno de esos niveles, tal y como lo indica el vector de color rojo en la figura.

Siguiendo la referencia de Labastida (2006), cuando plantea que "al formar conceptos, el hombre engendra, captura o concibe ideas: señala límites, marca fronteras y separa un objeto conceptual de otro" (Labastida, 2006, p. 11), sería necesario agregar que cuando se propone un concepto, una teoría y una opción metodológica se está posicionado una perspectiva ontológica particular, es decir, se está proyectando una noción específica de la realidad.

Un ejemplo de la importancia de lo ontológico en la definición de las propuestas de las ciencias sociales se puede identificar en Searle (1997) y su discusión con la perspectiva constructivista representada en la obra de Berger y Luckmann (2003). El punto central de diferencia y la tensión está en las concepciones ontológicas disímiles, como él lo plantea:

... nuestra investigación es ontológica, esto es, versa sobre el modo en que los hechos sociales existen, necesitamos una imagen del modo en que la realidad social casa con nuestra ontología general, del modo en que la existencia de hechos sociales se relaciona con otras cosas que existen. (Searle, 1997, p. 25)

La discusión no está en la búsqueda del génesis de conocimiento ${ }^{3}$, sino en la forma de comprensión de las propuestas de conocimiento. De igual manera, el interés de este texto no es defender una visión de realidad o una forma específica de conocer, sino mostrar que las tensiones epistemológicas y metodológicas están sustentadas en visiones ontológicas particulares. Esto quiere decir que, más que una propuesta explicativa del proceso de generación de conocimiento, se trata de un interés por brindar herramientas para entender las ciencias sociales en su diversidad.

La pretensión no es defender una perspectiva ontológica en particular, sino señalar que esta marcar la forma en la que se constituyen formas de trabajo en las ciencias sociales. En esta línea, es importante mencionar que

3 Para esto se puede consultar a García (2000), en este texto se puede encontrar una lúcida discusión sobre el proceso de construcción de conocimiento, edificada "sobre los hombros" de la propuesta de Jean Piaget. 
este trabajo se diferencia del amplio debate que se ha desarrollado en el marco de la filosofía analítica (Searle, 1997) y el realismo crítico (Archer, 2009; Bhaskar, 2005, Sayer, 2010), desde la cual se propone un abordaje en el marco de la denominada ontología social. Este trabajo no se ocupa de la ontología social, sino de la manera en lo que lo ontológico es asumido en las diversas corrientes de las ciencias sociales y la relevancia que esto tiene para comprenderlas.

Es necesario clarificar que la representación lineal, con una única lógica y dirección, se fundamenta en que se trata de un ejercicio meramente expositivo y propositivo que simplifica el entendimiento de la propuesta. Es claro que la lectura de todo proceso de conocimiento es dinámica y los avances en los niveles superiores pueden significar, a la postre, cambios en la perspectiva ontológica, lo que implica plantear interrelaciones. Esto es evidenciado por Houtart (2006), Wallerstein (2004 y 2006) y Maldonado (2014), cuando reflexionan sobre las implicaciones del trabajo del químico llya Prigogine respecto a la concepción de la realidad y la naturaleza. O cuando Roitman (2006) presenta las implicaciones del trabajo de los matemáticos Kurt Gödel y René Thom. Otra evidencia de esa complejidad en los componentes es la que propone Searle (1997) cuando establece la distinción entre lo epistémico y lo ontológico y su relación con lo objetivo y lo subjetivo.

La discusión que se propone queda retratada en la siguiente referencia a Ramos y Lamo (2006):

la descripción de la realidad social es, pues, inagotable y siempre inconclusa pues debería tomar en consideración todas las descripciones previas y, además, a sí misma. En razón de esto, la ciencia social es inevitablemente performativa, pues modifica la realidad al tiempo que hace su cartografía. (p. 235)

Frente a esto, se precisa que la propuesta está basada en entender las teorías y perspectivas metodológicas como elaboraciones situadas, propias de un contexto histórico, político y social. Con esta idea es posible esa lectura lineal, que va de una visión de realidad a una propuesta de las formas específicas de conocimiento. 
Lo anterior implica afirmar que, más que un modelo del comportamiento de la ciencia, se propone un punto de acceso que permite reconstruir las diversas perspectivas y la posición que estas tienen.

Como ya se mencionó, la discusión de este trabajo no será solamente de niveles y sentido, sino que se ejemplificará en las perspectivas antes mencionas.

\section{La ontología como puerta de acceso a las ciencias sociales}

Si en la actualidad se asume el propósito de entender las ciencias sociales, en los primeros esfuerzos se hará visible la diversidad de disciplinas, perspectivas, teorías, conceptos y nociones que esto implica, lo cual podría parecer abrumador. A esto se le puede denominar la complejidad de las ciencias sociales. Por esto se busca proporcionar una guía que permita un acceso a la comprensión de las propuestas y sus diferencias.

Lo que acá se pretende no es una discusión única y, menos aún, desvinculada de los múltiples esfuerzos que se han realizado, sino, por el contrario, se parte del reconocimiento de que a lo largo de la historia se han desarrollado diversidad de discusiones que buscan dar cuenta de la especificidad de las ciencias sociales. De la Garza y Leiva (2012) proponen que en la discusión sobre la metodología de las ciencias se han reflexionado y discutido cuatro tipos de relaciones, a saber: teoría e investigación empírica, teoría y las imágenes del mundo, teoría y preguntas normativas u orientadoras y saber, y la compresión teórica de la persona que investiga/ saber y la compresión de la persona lego.

En estas relaciones se busca identificar pautas que den sentido y permitan validar el trabajo en cada una de las disciplinas. En este artículo se coloca el acento en la segunda relación -teoría y las imágenes del mundo-, para dar sentido y contenido a las posiciones que se toman, de manera derivada, en las otras vinculaciones.

Además, en un contexto en el que, con mayor frecuencia, se discute la importancia de articulación entre áreas distintas del saber, surgen interrogantes sobre las formas de identificar puntos de encuentro y conexión. 
Como forma de ejemplificar otras respuestas a la necesidad de comprender el marco sentido y organización de las ciencias sociales, se pueden mencionar la noción del marco epistémico de García (2006) y retomada Amozurrutia (2012), o la de los paradigmas transversales o transdiciplinarios de Baraona et al.. (2015).

En el caso del marco epistémico, es posible identificar dos sentidos y usos. El primero refiere a una noción que sirve para lo siguiente:

... analizar los cambios que han tenido lugar históricamente en la ciencia, representa un sistema de pensamiento, rara vez explicitado, que permea las concepciones de la época en una cultura dada y condiciona el tipo de teorizaciones que van surgiendo en diversos campos del conocimiento. (García, 2000, p. 157)

Se trata de esa serie de ideas, posiciones y nociones que están en la base de las propuestas de conocimiento. La segunda, que se denomina marco epistémico común (García, 2000), la emplea para explicar las bases para el vínculo entre disciplinas, resaltando la correspondencia de los principios epistemológicos, como preocupación central para las condiciones de diálogo.

En el caso del paradigma común o transdisciplinario, se orienta a las bases de las nociones teóricas y metodológicas comunes, como plantea Baraona et al. (2016): "Entendemos por esta a cualquier paradigma transversal que cruza varias disciplinas específicas, se nutre de ellas; pero las rebasa y engloba al mismo tiempo con alguna propuesta teórica y metodológica" ( $p$. 18). El interés está en identificar las bases que dan sentido a la orientación de diversas disciplinas, para crear las condiciones de diálogo y construcción problemática conjunta.

Ambas implican posiciones en los niveles propuestos (Figura 1). En el caso de García (2000 y 2006), se basa en la discusión de la perspectiva epistemológica (tercer nivel en la figura). En el de Baraona et al. (2016), se asume que la discusión está en la posición del paradigma, y da énfasis, a lo que llama: "marco conceptual y metodológico", lo que vendría a ser una combinación de niveles, pero con un acento en lo metodológico (cuarto nivel). 
Aunque la lógica práctica de esas propuestas es buscar puentes entre disciplinas (más allá de las ciencias sociales), en este artículo se destaca el principio de comprensión, el cual se basa en la identificación de encuentros o correspondencia entre niveles de tensión en el marco de las ciencias y las humanidades.

En contraposición a las anteriores propuestas ${ }^{4}$, se plantea la comprensión de las perspectivas metodológicas desde las concepciones ontológicas. Argumentando que esta valoración permitirá identificar en encuentros y desencuentros entre las disciplinas y las formas de abordaje, pues, como plantean De la Garza y Leyva: “... la comprensión del método no podía disociarse de una determinada concepción de la realidad, ya sea sujeta a leyes universales o historizada, construida; reducida a la subjetividad o bien a la articulación sujeto objeto" (p. 24).

La propuesta se basa en que la concepción ontológica, en tanto posicionamiento sobre e/ ser, posibilita identificar la noción de la realidad, los acontecimientos y las relaciones, en las ciencias y las humanidades. La ontología es la noción básica sobre la que se edifica y desarrolla la delimitación de los campos de estudio y los intereses a lo interno de estos (objetos de estudio), los procedimientos y posibilidades de acceso al conocimiento (epistemología y metodología), y los valores que soportan la práctica del conocimiento (axiología).

De esta manera, el espacio básico para la comprensión (lo que implica la valoración sobre las posibilidades de vinculación) de las diferencias entre las ciencias naturales, las sociales y las humanidades, y la propia complejidad a lo interno de estos campos, puede ser accesible, si se trabaja, desde la base de las diferentes concepciones ontológicas que marcan la distancia o crean posibilidades de acercamiento.

En el siguiente apartado se presentará cómo la visión ontológica en dos perspectivas de las ciencias sociales permite determinar el posicionamiento en los niveles superiores de abstracción (Figura 1).

4 Una propuesta de organización de los paradigmas de las ciencias sociales desde la combinación de lo ontológico y lo epistemológico puede encontrarse en Tang (2011). Su texto es una referencia relevante, ya que muestra con claridad que la disputa de niveles para el abordaje de las ciencias sociales no es nueva, sino que hay múltiples referencias y posiciones.

Luis Diego Soto Kiewit 


\section{LA NOCIÓN ONTOLÓGICA EN LAS PERSPECTIVAS METODOLÓGICAS DE LAS CIENCIAS SOCIALES}

En las siguientes líneas se expone la perspectiva ontológica de dos corrientes metodológicas de las ciencias sociales. En cada uno de los casos se reconstruirá, desde diversas lecturas y propuestas, la perspectiva de realidad y cómo desde estas se edifican los niveles superiores, hasta llegar a una posición metodológica particular.

En este punto es necesario hacer dos aclaraciones: primero, la selección de ideas y posiciones es un ejercicio de interpretación, ya que no en todas las perspectivas se explicita claramente la noción de la realidad o la relación que esta tiene con las posiciones epistemológicas y metodológicas. Segundo, las perspectivas seleccionadas no son un ideario monolítico, que diversos autores e investigadores asumen y suscriben, sino que se trata de corrientes, es decir, trabajos y escritos que, aunque con distancias y tensiones, siguen una línea de reflexión.

\section{El posestructuralismo: una lectura desde la perspectiva ontológica}

En este subapartado se expone la concepción ontológica desde la que se edifica el posestructuralismo, para posteriormente derivar de ella una explicación de la propuesta de conocimiento desde la que opera y basa sus fundamentos.

La perspectiva ontológica de esta corriente se puede sintetizar en la siguiente referencia:

... la "realidad" es un producto que es constituido por un entrelazamiento de prácticas discursivas, poder y procesos cognitivos, los cuales a su vez determinan lo que puede ser percibido, pensando, experimentado y sentido como realidad. No existe una realidad independiente del discurso, dado que nuestra percepción sensorial y cognitiva siempre está mezclada discursivamente. (Moebius, 2012, p. 539)

La premisa básica es que la realidad se constituye en lo discursivo, como espacio de asignación de significados y sentidos. Lo social es el resultado de las relaciones entre las prácticas discursivas, el poder y los procesos 
cognitivos. Lo existente halla su explicación en los procesos de construcción de significados, que lo demarcan y definen.

Esto puede ser evidenciado en la obra de Laclau y Mouffe (2001):

... para que quepa una relación de representación hegemónica hay que definir su estatus ontológico. Y es en este punto donde la noción de lo social entendido como espacio discursivo (que posibilita relaciones de representación totalmente impensables en el seno de un paradigma fiscalista o naturalista) cobra una importancia extrema, (p. 15)

El discurso se constituye en el medio central de definición de lo social. Esta posición ontológica, afincada en lo discursivo, puede encontrarse en otras obras de posestructuralistas, aunque en algunos casos con ciertos matices, como es el caso de Foucault (2016) y Butler (2007).

Además, la realidad es un sistema abierto de múltiples interrelaciones, en donde se forman contextos específicos que toman sentido desde los discursos, a la vez que establecen sentidos y prácticas concretas. Plantea Butler (2007):

como una genealogía de la ontología del género, esta explicación tiene como objeto entender la producción discursiva que hace aceptable esa relación binaria y demostrar que algunas configuraciones culturales del género ocupan el lugar de "lo real" y refuerzan e incrementan su hegemonía a través de esa feliz autonaturalización. (p. 97)

La sociedad, las instituciones, las prácticas y los sujetos se explican desde los discursos que los constituyen, son estos los que definen lo "natural", "esperable" o "lógico" en un contexto específico5. Las formas y orientaciones en los diversos niveles son resultado de las prácticas (discursivas y no discursivas) $)^{6}$, las cuales no están preestablecidas, sino que son el re-

5 Foucault (2016) lo define en los siguientes términos: "Desde luego, si uno se sitúa en el nivel de una proposición, en el interior de un discurso, la separación entre lo verdadero y lo falso no es ni arbitraria, ni modificable, ni institucional, ni violenta" (p. 19).

6 Al menos en la propuesta de Foucault (2016) se hace la diferencia de prácticas discursivas y no discursivas, pues en otros trabajos, hay, más bien, una negativa a asumir esa dualidad, como por ejemplo en Laclau y Mouffe (2001). 
sultado de las relaciones entre formas discursivas, siempre contingentes. De forma concreta, se establece que "... la estructura discursiva no es una entidad meramente 'cognoscitiva' o 'contemplativa'; es una práctica articulatoria que constituye y organiza a las relaciones sociales" (Laclau y Mouffe, 2001, p. 132).

Continuando con las consecuencias de la noción ontológica de esta corriente de pensamiento, se presenta la concepción de sujeto desde la que se entiende lo social. Según Moebius (2012), en el posestructuralismo:

el sujeto no designa una unidad previa y que existiera por sí misma, sino un producto de una red de relaciones ocupada por el poder, entonces el proceso de devenir sujeto contiene un aspecto irreductible de vinculación a una dimensión externa, en la que él mismo no puede influir. (Moebius, 2012, p. 544)

El sujeto es el producto o resultado de las relaciones. No tiene un rol activo en la definición de lo social o lo cultural, sino que está supeditado a las relaciones constituidas a nivel estructural o institucional, lo cual implica que es un resultado de las relaciones de poder y los significados construidos socialmente, esto es válido para Foucault (2008, 2010 y 2016). Lo anterior se ejemplifica en la siguiente idea: "siempre que utilicemos la categoría 'sujeto', lo haremos en el sentido de 'posiciones de sujeto' en el seno de una estructura discursiva" (Laclau y Mouffe, 2001, p. 156) ${ }^{7}$.

El foco central de interés no son los sujetos, sino los discursos. Los agentes y sus acciones se explican como "posiciones diferenciales", en el contexto de los discursos que conforman la sociedad, en la búsqueda de crear opciones hegemónicas, es decir, "regularidad en la dispersión" (Laclau y Mouffe, 2001).

También se puede identificar apertura en otros trabajos de esta corriente, como en Butler (2007), cuando define su perspectiva como autora posestructuralista de la siguiente manera: "No estoy fuera del lenguaje que me estructura, pero tampoco estoy determinada por el lenguaje que hace

7 La noción de sujeto en el posestructuralismo no es una sola, sino que atraviesa algunas transiciones, como lo demuestra Vergalito (2007), buscando las influencias y posiciones en la obra de Ernesto Laclau. De manera que la definición brindada en este texto es solamente una deriva común de la perspectiva general de la corriente. 
posible este 'yo'. Este es el vínculo de auto-expresión, tal como lo entiendo" (p. 30).

Estos dos puntos de la noción ontológica del posestructuralismo (la visión de la realidad y los sujetos) permiten delinear el contorno general en el que se mueve esta corriente y sobre la que se construye una opción de conocimiento.

\section{La perspectiva posestructuralista y su enfoque}

En la perspectiva ontológica es posible enmarcar y extrapolar los principios con los cuales el posestructuralismo se posiciona en términos metodológicos.

Siguiendo el trabajo de Moebius (2012), se pueden identificar 4 principios básicos de la elaboración en esta corriente, a saber: la concepción de la temporalidad, la perspectiva de funcionamiento de los sistemas, el cuestionamiento al universalismo y la opción por la contingencia.

La concepción temporal se distancia del estructuralismo, hay una ruptura con esa visión centrada en la lectura sincrónica. En el posestructuralismo "la historia no puede ser vista como una continuidad del pasado y presente, ni como una separación de un 'antes' y un después'” (Moebius, 2012, p. 536). Esta posición implica un doble distanciamiento, por un lado, con la tradición centrada en la explicación histórica como proceso de desarrollo de lo social, y por otro, con la lectura estructuralista inicial. El resultado es una visión que combina ambos enfoques de temporalidad, sin absolutizarlos.

En este aspecto surge toda la tensión por la incorporación de la perspectiva histórica, que no estaba presente en el estructuralismo inicial. Esta opción por lo histórico como práctica explicativa puede verse en Foucault (2010), cuando posiciona su interés en el texto "Las palabras y las cosas":

... es más bien un estudio que se esfuerza por encontrar aquello a partir de lo cual han sido posibles conocimientos y teorías; según cuál espacio de orden del saber se ha constituido; sobre el fondo de qué a priori histórico y en qué elemento de positividad han podido aparecer las ideas, constituirse las ciencias, reflexionarse las 
experiencias en las filosofías, formarse las racionalidades para anularse o desvanecerse quizá pronto. (p. 15)

Esto será posteriormente desarrollado en la opción por la genealogía, como procedimiento de trabajo en sus obras. Esta posición la trata en el ensayo denominado: "Nietzsche, la genealogía, la historia". En cuanto al procedimiento de trabajo enfocado en el archivo histórico Dosse (2017b) plantea:

La pasión por el archivo va a llevar a Foucault a presentar expedientes históricos que le permitan poner en escena la forma en la que un cuerpo puede ser atrapado como apuesta del poder en el entrecruzamiento de múltiples dispositivos discursivos que se lo disputan. (p. 290)

Esa realidad constituida por lo discursivo no tiene sentido solamente en el plano de las relaciones actuales, sino que encuentra vinculaciones con el devenir y los diversos contextos históricos anteriores.

En la perspectiva de funcionamiento de los sistemas, las diversas elaboraciones teóricas posestructuralistas se distancian de las propuestas que plantean la diferenciación funcional como elemento organizativo y diferenciador de los sistemas. Frente a ellas ponen el acento en “... los procesos de desdiferenciación, las hibridaciones y la transgresión de fronteras de los códigos que trascienden los sistemas" (Moebius, 2012, p. 537). Estas ideas se interesan en las relaciones entre los diversos sistemas, ejemplificadas por el uso compartido de discursos, inclusive condiciones de saber que configuran sistemas, sus combinaciones o mutaciones necesarias (Foucault, 2010).

La posición anterior está sustentada en una visión ontológica concreta, en la que la realidad se entiende como hibridación, como interrelaciones que articulan realidades y condiciones diversas, en estructuras que están siempre abiertas, por lo que los traslapes y las influencias son una condición propia.

El cuestionamiento al universalismo se define como una crítica a las nociones que invitan a pensar condiciones predefinidas y naturales en lo social. Este principio se esgrime desde el cuestionamiento por las formas constitutivas que crean y definen esos universalismos, a la vez, que se ocupa de 
que queda al margen en las definiciones socialmente establecidas (Moebius, 2012). El universalismo es entendido como el interés de un discurso específico; una significación que se posiciona con mayor relevancia frente a otras. Esto es claro en Butler (2007).

Más que un discurso universal o una opción por una lógica de organización de lo social, se considera "... la apertura de lo social el fundamento constitutivo, la "esencia negativa" de lo existente, a los diversos "órdenes sociales' intentos precarios y, en última instancia, fallidos, de domesticar el campo de las diferencias" (Laclau y Mouffe, 2001, p. 132). Lo social es el resultado de las relaciones en diversos planos, sin una estructura preestablecida.

En otras palabras, se puede plantear:

[En esta corriente] las retóricas "finalistas", teleológicas o el propio "fin de la historia" son tentativas siempre ineficaces de domesticar lo indomesticable. Son ineficaces desde el punto de vista lógico porque buscan pensar al régimen político desde el punto de vista óntico exclusivamente, no teniendo en cuenta o simplemente despreciando toda ontología de lo político, la cual es inestable y abierta. (Groppo y De Mendonça, 2010, p. 15)

Un elemento destacable de la idea anterior es que la referencia crítica a las aproximaciones universalistas está constituida desde lo ontológico, en tanto referencia y punto de definición de una lógica de lo social.

La opción por la contingencia en esta corriente se rescata en la importancia que está condición tiene en lo social. Esto, porque los significados, los discursos y los fenómenos no tienen una forma preestablecida (como se planteó en el punto anterior), sino que las interrelaciones establecen el curso de los acontecimientos. Los discursos se producen y reproducen a partir del dinamismo de los sistemas sociales, y desde esta relación configuran formas organizativas (siempre abiertas a la transformación).

Esa opción por la lectura de lo contingente como elemento característico de lo social es claramente expuesta en la obra de Butler (2007): 
Revelar los actos contingentes que crean la apariencia de una necesidad naturalista ... es un trabajo que ahora asume la carga adicional de enseñar cómo la noción misma del sujeto, inteligible sólo por su apariencia de género, permite opciones que antes habían quedado relegadas forzosamente por las diferentes reificaciones del género que han constituido sus ontologías contingentes. (Butler, 2007, p. 98)

Todos estos principios están en correspondencia con la opción ontológica, ya que son una derivación lógica de la forma en la que se entiende la sociedad. No es posible distanciar ambos niveles, la visión de realidad está articulada con la definición del procedimiento para su conocimiento.

El método se supedita a la perspectiva de la realidad, al interés que cada autor o autora de esta corriente tiene. El problema no es la discusión del método, sino de la forma de explicar una realidad específica, que se constituye y toma sentido en lo discursivo. Como lo expresa el siguiente comentario de Foucault (2013):

No tengo un método que aplique de la misma forma a dominios deferentes. Al contrario, diría que es un mismo campo de objetos, un dominio de objetos el que trato de aislar utilizando instrumentos que encuentro o forjo, en el momento mismo de hacer mi investigación, pero sin privilegiar en absoluto el problema del método. (p. 73)

\section{El estructuralismo constructivista: una lectura desde la perspectiva ontológica}

Si tuviese que caracterizar mi trabajo en dos palabras, es decir, como se hace mucho hoy, aplicarle una etiqueta, hablaría de constructivist structuralism o de structuralist constructivism, tomando la palabra estructuralismo en un sentido muy diferente de aquel que le da la tradición saussuriana o lévi-straussiana.

(Bourdieu, 2000, p. 127)

El epígrafe anterior muestra la clasificación de Bourdieu sobre su obra. Aunque lo hace con alguna resistencia, se puede señalar que no hay una distinción entre las formas de clasificación, sino que su uso las coloca en un mismo nivel. Esto, como se va a proponer en este artículo, es impreciso, 
ya que en las corrientes de trabajo hay acentos (y especialmente en su obra), en uno u otro polo, que marcan la forma de abordaje de la realidad.

La manera más clara de entender la concepción que el autor tiene es la siguiente referencia:

... con "estructuralismo" o "estructuralista" pretendo decir que en el mundo social mismo existen ... estructuras objetivas independientes de la conciencia y de la voluntad de los agentes, las cuales son capaces de orientar o de restringir las prácticas o representaciones de ellos. Con "constructivismo", pretendo decir que hay una génesis social de los esquemas de percepción, de pensamiento y de acción que son constitutivos de lo que denomino habitus; y, por otra parte, la hay de las estructuras sociales, y en especial de lo que denomino campos. (Bourdieu, 2000, p. 127)

El autor pasa de denominar a definir. Establece que su concepción encuentra la explicación de lo social en la interacción de dos niveles (el estructural y el de los esquemas de pensamiento).

Esa posición implica un diálogo crítico con las corrientes más elementales de la compresión del mundo social (en la tradición sociológica). Las corrientes pueden calificar como objetivistas y subjetivistas. Las primeras son aquellas que visualizan la sociedad como resultado de la producción de las estructuras (o sistemas), y, las segundas las que defienden que lo social se construye en las acciones e interacciones de los actores.

La posición de Bourdieu es una salida a esa dicotomía, pues implica la unificación de concepciones. Es una síntesis que, en lugar de posicionar uno de los dos planos, los une. La clave de este vínculo es la identificación de una relación dialéctica de complementariedad ${ }^{8}$ entre ambos planos de la realidad (Giménez, 2002).

Una consideración de relevancia es la tensión que guarda la denominación de constructivismo estructural ${ }^{9}$, con la que se ha reconocido y ubicado su

8 Como plantean diversos estudios (Giménez, 2002; Beytía, 2012), esta acepción de la dialéctica es tomada por Bourdieu de la obra de Bachelard.

9 Como bien señala Álvarez (1996), esta no es la única dominación que ha tenido esta corriente, ya que a esa doble tensión se puede sumar nociones como: estructuralismo crítico y estructuralismo genético. 
trabajo $^{10}$. Lo anterior porque ha recibido importantes críticas e interpretaciones diversas. En lo que respecta a la presente reflexión hay una toma de distancia, pues, como propone Corcuff (2015), la aproximación a Bourdieu permite ver una lectura que hace transición entre las estructuras y las interacciones (con ese orden de prioridad y lógica), es decir, lo que se debería llamar un estructuralismo constructivista ${ }^{11}$.

En este punto surge la pregunta: ¿Cuál es la concepción ontológica en la que se soporta esta corriente de pensamiento?, pues Bourdieu siempre fue enfático en que la teoría solo podía ser pensada desde un diálogo con la realidad. Es decir, construida en un vínculo directo y desarrollada a partir de interacción constante (Bourdieu, Chamboredon, y Passeron, 2008 y Bourdieu y Wacquant, 2005).

\section{La visión ontológica del estructuralismo constructivista}

En esta corriente es posible reconstruir elementos de la posición ontológica en la que Bourdieu basa su perspectiva, procedimientos y conceptos ${ }^{12}$. Este apartado se enfoca en identificar, en su obra, así como en algunos de los análisis realizados por otros autores, esa visión del ser que permite organizar su propuesta de conocimiento.

Una forma de acercarse a la visión ontológica es identificar cómo Bourdieu visualiza el mundo. La forma en la que concibe el ser de las cosas en la sociedad (la manera en que define lo que se visualiza como "dado" o "natural"). Con esta finalidad se emplea la siguiente referencia:

Se puede representar así al mundo social en forma de espacio (de varias dimensiones) construido sobre la base de principios

10 Aunque se trata de la obra de un solo autor, por las implicaciones de sus ideas, conceptos y formas de trabajo, se le ubica como una corriente de pensamiento. Son amplias las aportaciones que brindó a las ciencias sociales, además de que no puede dudarse la vigencia de su pensamiento en la discusión actual de las ciencias sociales.

11 Esta fórmula de conocimiento, construida desde la tensión en entre las estructuras y las relaciones, no es exclusiva de su obra, sino que, también se puede ubicar en el trabajo de autores como Archer (2009), Giddens (2006), Rubinstein, (2001) y Elias (1990, 1991). Incluso, en este espacio se puede agregar la obra de Berger y Luckmman $(1997,2003)$, aunque, en el caso de estos, el acento está en las interacciones.

12 En el trabajo de Archer (2009) se puede ubicar una reflexión que expone y detalla las concepciones ontológicas disimiles en las teorías conflacionistas y no conflacionistas, como elemento central de la divergencia. 
de diferenciación o distribución constituidos por el conjunto de las propiedades que actúan en el universo social en cuestión, es decir, las propiedades capaces de conferir a quien las posea con fuerza, poder, en ese universo. Los agentes y grupos de agentes se definen entonces por sus posiciones relativas en ese espacio (el resaltado es del original) (Bourdieu, 1989, pp. 281-282).

Según esta referencia, la realidad es un espacio de múltiples dimensiones que se entrecruzan y relacionan. Es una totalidad compleja (Marqués, 2006), la cual configura los campos que organizan lo social y demarcan el habitus. Es el espacio en que los agentes encuentran posibilidades de acción y, a partir de los cuales, establecen su sentido práctico (que no es solamente reproductivo, sino también productivo).

Se puede derivar también que, para esta corriente, la realidad no es resultado de condiciones sustanciales o de contenido preestablecido, sino que: "... concibe las instituciones... de manera relacional, como configuraciones de relaciones entre agentes individuales y colectivos" (Corcuff, 2015, p. 44). El enfoque de relaciones es coincidente con la corriente estructuralista, la diferencia estriba en la capacidad de los agentes de tener un rol activo en el proceso y la relevancia que se le confiere lectura histórica ${ }^{13}$.

En relación con este rol activo de los agentes en el proceso, Corcuff (2015) extrae del texto: "Meditaciones Pascalinas", cuatro principios de la concepción antropológica de Bourdieu. Estos son: la lógica de lucha contra la muerte simbólica, la lógica de los intereses, la lógica de la primacía de un cuerpo humano no reflexivo y la lógica de la libertad relativa merced al conocimiento de los determinismos sociales. Estas lógicas demarcan la idea de un agente que lucha por la existencia simbólica, tiene intereses (en el marco de los campos en los que participa), se relaciona primero con el cuerpo y luego de manera reflexiva y es producto de un doble juego de determinación (interna y externa).

Estas ideas marcan la visión de "ser humano" presente en sus análisis y permite explicar la forma de abordaje. Como puede visualizarse en la siguiente afirmación de Bourdieu (1989):

13 Como señala Álvarez (1996) en referencia a la concepción de Bourdieu "La forma del espacio social no es la misma a lo largo de toda la historia ni de todas las sociedades, sino que la forma que adopta depende de la distribución adoptada dentro de cada campo" (p.148). 
La posición de un agente determinado en el espacio social puede definirse entonces por la posición que ocupa en los diferentes campos, es decir, en la distribución de los poderes que actúan en cada uno de ellos; estos poderes son ante todo el capital económico -en sus diversas especies-, el capital cultural y el social, así como el capital simbólico, comúnmente llamado prestigio. reputación. renombre, etcétera, que es la forma percibida y reconocida como legitima de estas diferentes especies de capital. (p. 283)

En concordancia con los principios antropológicos antes establecidos, esa referencia deja ver la lógica de posición desde los campos en los que el agente se define y tiene sentido. Así también, esa lucha por acceso a los capitales que tienen relevancia en cada uno de esos espacios, ya sean sociales o institucionales.

De esta concepción ontológica se derivan una serie de ideas en la corriente estructuralista constructivista, las cuales se ven reflejadas en su obra. Como por ejemplo en la concepción de la distinción (Bourdieu, 1998) o su teoría de clases (Álvarez, 1996).

El siguiente nivel en el desarrollo de la reflexión invita a plantear estas preguntas: ¿Qué implicaciones tiene esta visión ontológica en la práctica de conocimiento? Para ser más precisos: ¿Cuáles son las implicaciones epistemológicas y metodológicas que resultan de esta visión de realidad?

\section{La perspectiva epistemológica y metodológica en el estructuralismo constructivista}

Una vez demarcada la definición de la corriente y sus bases ontológicas, se establecen los elementos epistemológicos y metodológicos que resultan de estas, con el objetivo de establecer vínculos de coherencia y organización del estructuralismo constructivista en esos otros planos.

A nivel epistemológico, Beytía (2012) establece que la propuesta estructural constructivista tiene tres pilares principales. A saber: la filosofía de la ciencia relacional, la filosofía de la acción disposicional y la sociología reflexiva. Cada uno de estos marcan preceptos desde los cuales se organiza la forma de acceso al conocimiento en esta corriente. 
La filosofía de la ciencia relacional está basada en la concepción de una realidad que tiene sentido en el marco de las relaciones, ya que la explicación de lo social debe encontrarse en las configuraciones de las relaciones.

En el tanto se plantea esta condición propiamente epistemológica, es necesario considerar que "... el principio de relacionalidad es utilizado doblemente por Bourdieu, tanto para el entendimiento de los fenómenos sociales como para la creación de su propio sistema conceptual" (Beytía, 2012, p. 16). A esto se podría agregar que incluso está en sus procedimientos metodológicos (Baranger, 2004).

La filosofía de la acción disposicional está ligada a la concepción de agente. Lo que propone es que estos no agencian desde la libertad total, sino que sus acciones tienen sentido a partir de los campos (nivel estructural) y las formas de habitus. Es decir, en el marco de una serie de disposiciones establecidas (sin negar las posibilidades de transformación).

La sociología reflexiva interpela directamente a la persona que investiga como agente que se mueve y adquiere sentido en campos específicos. Según lo establece Beytía (2012) “... el investigador debe estar consciente de que su observación es condicionada por su origen y sus características sociales" (p. 20). Esto recuerda el principio de la vigilancia epistemológica, que ya estaba presente desde "El oficio del sociólogo" (Bourdieu et al.. 2008).

En cada uno de los principios es posible establecer una relación directa con la concepción ontológica, ya que esa visión de realidad demarca la lógica de construcción conceptual y epistémica de su trabajo. La manera en la que se entiende la realidad delimita las estrategias para su conocimiento, esto es patente en su obra.

A nivel del procedimiento más metodológico, el trabajo de esta corriente se basa en la definición de dos momentos: el objetivista y subjetivista. Cada uno de ellos está directamente articulado a esa concepción ontológica, desde la que se estable la condición de posibilidad de lo social en la interacción de los dos espacios de relaciones (el estructural y el de los esquemas de percepción y pensamiento). Como plantea Capdeviella (2012): "Bourdieu se opone a todas las formas de monismo metodológico 
que conlleva aseverar la prioridad ontológica de la estructura o el agente, del sistema o del actor, de lo colectivo o lo individual ..." (p.3).

El uso del procedimiento por medio de esos dos momentos puede encontrarse en la siguiente cita a Bourdieu (2000):

Por un lado, las estructuras objetivas que construye el sociólogo en el momento objetivista, al apartar las representaciones subjetivas de los agentes, son el fundamento de las representaciones subjetivas y constituyen las coacciones estructurales que pesan sobre las interacciones; pero, por otro lado, esas representaciones también deben ser consideradas si se quiere dar cuenta especialmente de las luchas cotidianas, individuales o colectivas, que tienden a transformar o a conservar esas estructuras. (Bourdieu, 2000, p. 129)

Es evidente que su trabajo se articula con la concepción estructuralista constructivista. En términos de procedimiento, demarca una forma de trabajo que busca acceder a los dos ámbitos, se trata de una lectura de la realidad desde la complementariedad de sus dos niveles constitutivos.

Este doble esfuerzo de abordaje es posible reconocerlo en sus trabajos de investigación. Un claro ejemplo de ello está en el texto "La distinción". Además, por la naturaleza diversa de su obra, estas valoraciones más epistémicas y metodológicas ${ }^{14}$ pueden ser ubicadas en textos como "El Oficio del sociólogo", "Una invitación a la sociología reflexiva" y "Cosas dichas".

Su concepción dialéctica de la complementariedad está presente en todos los niveles, desde el ontológico hasta el metodológico. Esta es una clave de gran relevancia para entender sus obras y las diversas posiciones que desde esta corriente se asumen, ya que está en la base de ese doble vínculo que caracteriza su trabajo.

14 En la obra de Baranger (2004) se puede encontrar una detallada reflexión de la relación entre las concepciones teóricas del autor y su perspectiva del trabajo más instrumental. Como son su visión del dato y sus procedimientos de trabajo. 


\section{CONCLUSIONES}

Las discusiones sobre las perspectivas metodológicas de las ciencias sociales han significado el desarrollo de una amplia variedad de reflexiones, en las cuales se da cuenta de la particularidad de cada uno de los abordajes, y se han mostrado las tensiones que implican y las congruencias que tienen. El acento ha estado en hacer visible dos niveles del posicionamiento, a saber: el epistemológico y el metodológico.

En contrapunto a esas formas de acercamiento, en este artículo se propone que las concepciones ontológicas son el parámetro desde el cual se construyen las corrientes, defendiendo la idea de que estas nociones son la base desde la que se articulan todas las perspectivas. Como se muestra en la figura inicial, la visión ontológica transforma los demás niveles de definición en las ciencias sociales, a saber: el axiológico, la epistemológico y el metodológico.

Los ejemplos utilizados permiten evidenciar la plausibilidad de esta propuesta, pues, en ambos casos, en el posestructuralismo y el estructuralismo constructivista, se logró establecer una lectura de la propuesta metodológica y epistemológica desde las concepciones ontológicas.

En el caso del posestructuralismo, a pesar las tensiones a lo interno de la corriente, es posible ubicar puntos comunes en la diversidad de autorías, como se mostró en el desarrollo del artículo. Sobre la perspectiva ontológica, coindicen en entender la realidad como resultado de las relaciones discursivas. Esta primacía del discurso se refleja en la noción de sujeto supeditada a los discursos. Esto implica la visión de un sujeto que actúa y toma sentido en el marco de las diversas formaciones discursivas (a modo de posiciones).

De esa visión ontológica se deslindan cuatro puntos centrales de la opción posestructuralista: la concepción de la temporalidad, la perspectiva de funcionamiento de los sistemas, el cuestionamiento al universalismo y la opción por la contingencia.

En el caso del estructuralismo constructivista, la realidad es un espacio conformado por múltiples dimensiones relacionadas por principios de diferenciación o distribución. A su vez, la visión antropológica tiene cuatro 
principios: la lógica de lucha contra la muerte simbólica, la lógica de los intereses, la lógica de la primacía de un cuerpo humano no reflexivo y la lógica de la libertad relativa, merced al conocimiento de los determinismos sociales. Estos principios demarcan la idea de un agente que lucha por la existencia simbólica, tiene intereses (en el marco de los campos en los que participa), se relaciona primero con el cuerpo y luego con la reflexión, y es producto de un doble juego de determinación (interna y externa).

Desde esos principios es posible establecer la opción epistemológica y metodológica que se construye en el estructuralismo constructivista, la cual permite identificar el tipo de intereses de conocimiento y la forma en la que se trabaja (los procedimientos específicos de investigación). En términos de procedimiento, demarca una forma de trabajo que busca acceder a los dos ámbitos, se trata de una lectura desde la complementariedad de sus dos niveles constitutivos.

Para cerrar y en congruencia con la organización propuesta, es importante derivar las siguientes ideas:

Las concepciones ontológicas pueden ser la "puerta" de acceso a la polifonía de perspectivas y teorías de las ciencias sociales. Esta forma de identificación, centrada en la visión de realidad, es de utilidad para marcar las diferencias de criterio y orientación, lo cual ayudaría a la compresión de las ciencias sociales.

El marco ontológico puede servir de base para la discusión en los procesos de construcción de conocimiento en los trabajos entre áreas de conocimiento diferenciadas, independientemente del nivel al que pretendan llegar (multidisciplinaredad, interdisciplinariedad o transdisciplinariedad). Iniciar con la clarificación de la visión de realidad puede contribuir a generar acuerdos sobre los cuales definir los parámetros de relación para, de esa manera, lograr un diálogo más fluido en los niveles axiológico, epistemológico y metodológico. 


\section{AGRADECIMIENTOS}

En la elaboración de una reflexión como esta es importante reconocer los aportes y comentarios de las personas contribuyeron a su elaboración. Aunque no figuran en la autoría, sus ideas forman parte de la discusión y contribuyeron a mejorar el texto. En específico quiero agradecer los aportes de Alexis Segura Jiménez y de las personas que evaluaron el manuscrito, sus comentarios fueron de gran relevancia para depurar el artículo, demostraron no solamente una lectura atenta, sino también un criterio versado en el campo de discusión.

\section{REFERENCIAS}

Álvarez, A. (1996). El constructivismo estructuralista: La teoría de las clases sociales de Pierre Bourdieu. Reis: Revista española de investigaciones sociológicas, 75, 145-172.

Amozurrutia, J. (2012). Complejidad y sistemas sociales: Un modelo adaptativo para la investigación interdisciplinaria. UNAM, Centro de Investigaciones Interdisciplinarias en Ciencias y Humanidades. Colección Debate y reflexión.

Archer, M. (2009). Teoría social realista: El enfoque morfogenético (Traducción de Daniel Chernilo). Ediciones Universidad Alberto Hurtado.

Baraona, M., Chaves, I., Esquivel, E., Gutiérrez, M., Muñoz, D., Rincón, L., Barquero, J., Chuprime, A., Goméz, J., Mora, J., Pérez, L., Rojas, S. y Sancho, M. (2016). La Cátedra de Rolando García de humanismo, interdisciplina y complejidad del CEG-UNA. Revista Nuevo Humanismo, 4(2), 7-14, http://dx.doi.org/10.15359/rnh.4-2.1

Baranger, D. (2004). Epistemología y metodología en la obra de Pierre Bourdieu. Prometeo Libros Editorial.

Bhaskar, R. (2005). The Possibility of Naturalism: A Philosophical Critique of the Contemporary Human Sciences. Routledge.

Berger, P. y Luckmann, T. (2003). La construcción social de la realidad (Traducción de Silvia Zuleta). Amorrortu. 
Berger, P. y Luckmann, T. (1997). Modernidad, pluralismo y crisis de sentido (Traducción Centro de Estudios Públicos). Paidós

Beytía, P. (2012). Una lectura bourdieuana acerca de Bourdieu: La posición epistemológica del constructivismo estructuralista. Revista Persona y Sociedad, . 26(3), 1-32.

Bourdieu, P. (1989). El espacio social y la génesis de las "clases". Estudios sobre las culturas contemporáneas, 3(7), 27-55.

Bourdieu, P. (1998). La distinción: Criterio y bases sociales del gusto (Traducción de María del Carmen Ruiz). Editorial Taurus.

Bourdieu, P. (2000). Cosas dichas (Traducción de Margarita Mizraji). Gedisa.

Bourdieu, P., Chamboredon, J. y Passeron, J. (2008). El oficio de sociólogo. Presupuestos epistemológicos. Editorial Siglo XXI.

Bourdieu, P. y Wacquant, L. (2005). Una invitación a la sociología reflexiva (Traducción de Ariel Dilon). Editorial Siglo XXI.

Butler, J. (2007). El género en disputa: El feminismo y la subversión de la identidad (Traducción de María del Mar García). Ediciones Paidós Ibérica.

Capdevielle, J. (2012). La sociología figuracional de Norbert Elías y el estructuralismo genético de Pierre Bourdieu: Encuentros y desencuentros. Aposta. Revista de Ciencias Sociales, 52, 1-23.

Corcuff, P. (2015). Las nuevas sociologías. Principales corrientes y debates (Traducción de Luciano Padilla). Editorial Siglo XXI.

De la Garza, E., y Leyva, G. (2012). Tratado de metodología de las ciencias sociales: Perspectivas actuales. Universidad Autónoma Metropolitana y Fondo de Cultura Económica.

Dogan, M. (1997a). Las nuevas ciencias sociales: Grietas en las murallas de las disciplinas. Revista Internacional de Ciencias Sociales, 153. 
Dogan, M. (1997b). ¿Interdisciplinas? Revista Relaciones, 157, 16-18.

Dosse, F. (2017a). Historia del estructuralismo. Tomo I: El campo del signo, 1945-1966 (Traducción de María del Mar García). Editorial AKAL.

Dosse, F. (2017b). Historia del estructuralismo. Tomo II: El canto del cisne, 1967 hasta nuestros días (Traducción de María del Mar García). Editorial AKAL.

Elias, N. (1990). Compromiso y distanciamiento: Ensayos de sociología del conocimiento (Traducción de José Antonio Alemany). Ediciones Península.

Elias, N. (1991). Mozart. Sociología de un genio (Traducción de Marta Fernández y Oliver Strunk). Ediciones Península.

Foucault, M. (2008). Vigilar y castigar: Nacimiento de la prisión (Traducción de Aurelio Garzón del Camino). Editorial Siglo XXI.

Foucault, M. (2010). Las palabras y las cosas: Arqueología de las ciencias humanas (Traducción de Aurelio Garzón del Camino). Editorial Siglo $\mathrm{XXI}$.

Foucault, M. (2013). El poder, una bestia magnifica: Sobre el poder, la prisión y la vida (Traducción de Horacio Pons). Editorial Siglo XXI.

Foucault, M. (2016). El orden del discurso (Traducción de Alberto González Troyano, 1. ${ }^{\text {era }}$ ed.). Fábula Tusquets Editores.

Francés, M. (2005). Socialización, educación y reproducción cultural: Bourdieu y Bernstein. Revista interuniversitaria de formación del profesorado, 19(1), 159-174.

García, R. (2000). El conocimiento en construcción: de las formulaciones de Jean Piaget a la teoría de sistemas complejos. Gedisa.

García, R. (2006). Sistemas complejos. Gedisa. 
Giddens, A. (2006). La constitución de la sociedad: Bases para la teoría de la estructuración (Traducción de José Luis Etcheverry). Amorroutou Editores.

Giménez, G. (2002). Introducción a la sociología de Pierre Bourdieu. Colección pedagógica universitaria, 35-38, 1-11.

González, P. y Roitman, M. (Eds.). (2006). La formación de conceptos en ciencias y humanidades. Editorial Siglo XXI.

Groppo, A. y De Mendonça, D. (2010). Postestructuralismo y política. Revista Pensamiento Plural, 7, 11-19.

Harré, R., \& Madden, E. (1998). Conceptual and natural necessity. In Archer, M., Bhaskar, R., Collier, A. Lawson, T. \& Norrie, A. (Eds.), Critical Realism: Essential Readings. Routledge.

Labastida, J. (2006). Prologo. El problema del concepto. En P. González y M. Roitman (Eds.), La formación de conceptos en ciencias y humanidades. Editorial Siglo XXI.

Laclau, E., y Mouffe, C. (2001). Hegemonía y estrategia socialista. Fondo de Cultura Económica.

Houtart, F. (2006). La ética de la incertidumbre en las ciencias sociales. Editorial CLACSO.

Kuhn, T. (1996). La estructura de las revoluciones científicas. Fondo de Cultura Económica.

Maldonado, C. (2014). ¿Qué es un sistema complejo? Revista colombiana de filosofía de la ciencia, 14(29), 71-93.

Marqués, I. (2006). Bourdieu o el «caballo de Troya» del estructuralismo. Revista Española de Investigaciones Sociológicas (REIS), 115(1), 69-100.

Moebius, S. (2012). Posestructuralismo y ciencias sociales (Traducción de Marco Romano Hassán) En Tratado de metodología de las ciencias 
sociales: Perspectivas actuales, (pp. 522-566). Fondo de Cultura Económica

Ramos, R. y Lamo, E. (2006). Por un diálogo interdisciplinario: Un axioma y dos teoremas de la ciencia social. En González, P. y Roitman, M. (Eds.), La formación de conceptos en ciencias y humanidades. Editorial Siglo XXI.

Rotiman, M. (2006). Ciencias de la certidumbre y ciencias de la incertidumbre. En P. González y M. Roitman (Eds.), La formación de conceptos en ciencias y humanidades. Editorial Siglo XXI, México.

Rubinstein, D. (2001). Culture, structure, and agency: Toward a truly multidimensional sociology. Pine Forge Press.

Sayer, A. (2010). Method in Social Science: A Realist Approach. Routledge.

Searle, J. (1997). La construcción de la realidad social. Paidós.

Tang, S. (2011). Foundational paradigms of social sciences. Philosophy of the Social Sciences, 41(2), 211-249.

Vergalito, E. (2007). Postestructuralismo y sujeto: Reflexionando desde Laclau. 4 Jornadas de Jóvenes Investigadores Instituto de Investigaciones Gino Germani, Facultad de Ciencias Sociales, Universidad de Buenos Aires. https://www.aacademica.org/000-024/211.

Wallerstein, I. (2004). Las incertidumbres del saber. Editorial Gedisa.

Wallerstein, I. (Ed.). (2006). Abrir las ciencias sociales: Informe de la Comisión Gulbenkian para la reestructuración de las Ciencias Sociales. Editorial Siglo XXI. 
